\title{
Espondilodiscitis y artritis de tobillo por Candida albicans en escolar
}

\author{
Luis Hernån González I. ' ; Elba Wu $\mathrm{H}_{.}^{1}$; Marta Miranda $\Lambda .^{\prime}$; \\ Patricio Rojas A.

\section{Candida albicans osteoarthritis and spondilodyscitis in chronic mucocutaneous candidiasis}

\begin{abstract}
A thirteen year ofd boy with known mucocutaneous candidiasis since age six month, had evidence of arthritis at his right ankle together with lower thoraxic pain $u$ l xyphosis. At $K$ ray examination right ankle's articular space was disminished and an initially eroded upper surface of the astragallus was seen, together with evidence of imparted T10-T17 vertebral bodies and spondilodyscitis at T10-11 and T4-5. Triphasic oseous scintigraphy showed increased counts in the afected ankle and in 711 vertebral body. Candida albicans was isolated from articular bioss but no microorganism was identitied trom T11 samples. During the search,of an immunologic system abnormality. malignant tumors and HIV were discarded, but selective deficiency of celular immunity for Candida albicans was demonstrated. He was treated with anphotericin B and 5-1fuocytocin for along two months approximately, physiotherapy and oral ketoconazole for an additional six months, without complications. He is now asymptomatic. without evidence of osteoarticuiar infection. There it is only slight equinus deformity at his right foot and minimal non painful dorsal xyphosis.

(Key words: candidiasis, mucocutaneous, asteoarticular, spondilodyscitis.)
\end{abstract}

Las infecciones mayores por hongos, en particular por Candida $s p$., se asocian, por lo general, a factores predisponentes como terapia antimicrobiana de amplio espectro, alimentación pa. renteral, catéteres venosos y arteriales, diabetes mellitus, adicción a opiáceos, administración de esteruides, déficit de mieloperoxidasa, prótesis valvulares, inmunosupresión, trasplante de órganos y enfermedades neoplásicas. Entre los niños con mayor riesgo figuran los recién nacidos que reciben antibióticos de amplio espectro, especialmente si son prematuros extremos, y los tratados por enfermedades neoplásicas ${ }^{1-5}$. A toda edad las infecciones mayores por Candida pueden ubicarse en cualquier parénquima, incluso el globo ocular: sin cmbargo, el compromiso osteoarticular sólo representa 1 a 2 por ciento de los casos de infecciones diseminadas $^{3}$ y muestra preferencia por el esqueleto axial (especialmente los espacios intervertebra-

1. Departamento y Servicio de Pediatría. Hospital San Juan de Dios.

2. Servicio de Ortopedia y Traumatología Infantil, Hospital San Borja-Arriarán. les), segujdo del carpo, fémur, articulaciones costocondrales y escápulo humeral ${ }^{2,7,8}, \mathrm{Se}$ describe un caso de candidiasis mucocutánea con compromiso periférico y axial en un escolar de 13 años de edad.

\section{Caso clínico}

Varón de 13 aftos de edad en la actualidad, hijo único de padres consanguíneos (primos). Gestación y período de recièn naciơo sáno, peso de nacimiento 4700 g. Lactuncia materna exclusiva los primeros dos meses de vida, inmunización completa, crecimiento y desarrollo psicomotor normales. Desde los seis meses hasta los cuatro años sutrió aigorra bucal persistente. a pesar de múltiples tratamientos locales y sistémicos con antimicóticos $y$ desde los cuatso años y hasta el momento de su ingieso, onicomicosis. A los cuatio años de cdad tuvo sarampión que evolucionó sin complicaciones. En marzo de 1987 sufrió traumatismo de tobillo derecho con aumento de volumen doloroso que no cedió con antinhlamatorios no esteroidales, reposo e inmovilización. La radiografía de la zona, en octubre de ese año, revelaba aumento de partes blandas, osteoporosis difusa, disminución del espacio tibiotarsiano y erosión de la superficje articular del astrágalo (figura 1). Una biopsia del tobillo derecho, en enero de 1988 , mostró sinovitis crónica fibrinoleucocitaria, granulomatosi con abundantes pseudomicelios de hongos; 


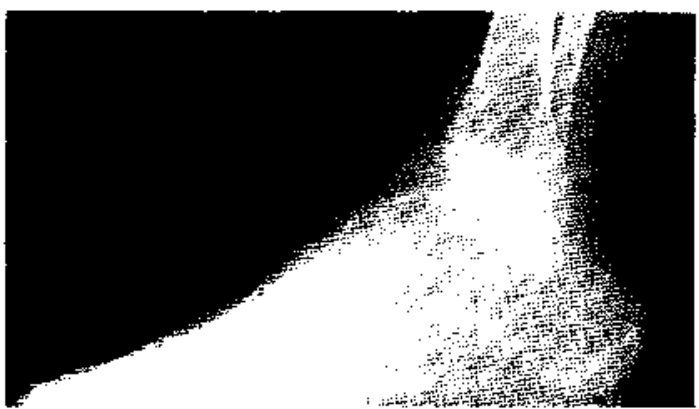

Figura 1: Radioñafía de pie, lateral, mostrando gran ostcoporosis difusa, cón disлinución del espacio tibiotarsano e irregularidad erosiva de la superficie asticular del istrágalo.

otra, en marzo del mismo año, mostraba levaduras en al cxamen ditecto y Caridicta albicans en el cultivo, la que tambión se obtuvo de las uñas de manos. En marzo de 1988 ingrewó al hoxpital con signos de artritis del tohillo derecho $y$ xifosic angulada dolorosa de la columna dorsal bajá. Fl cintigrama óseo trifásico mostró que habia mayor captación en el tobillo afectado $y$ el cuerpo vertebral de T11 (figura 2). En la radiografía de columina se encontraton signos de espondilodiscitis entre tas vértebras TlO y T11, impactación de los cuerpos vertebrales y lesión inicjal de T4 y T5 (figuras 3, 4). En la electrotoresis de proteínas plasmáticas mostraba disminución relativa de albúmina $y$ aumento absoluto de globulina $\alpha 1 y \alpha 2$. Inmunnelectrotoresis de intrunoglobulinux. complemento (C.3), subpoblaciones liniocitarias, número y proporción de l.T4 y LT8 e indice fagocítico eran nomales para nuestro laboratorio, pero el porcentaje de muerte celular estaba aumentado. La reacción de hipersensibilidad retardada y provocación con 24-dunitroclorobenceno eran posituvas. La prueba múltiple CMT (R) de hipersensibjlidad cutánea y la búsquedal de candidina circulante (Ig ant $\mathrm{j} \cdot$ Comlida abicans) fucron repetidamente negativas. Fue tratado con anfotericina $\mathrm{B}(0,6 \mathrm{mg} \cdot \mathrm{kg} \cdot \mathrm{d}$ ia hasta completar aproximadimente $1500 \mathrm{mg}$ ), aso. ciada a 5-fluorcitosina (200 mg dia) durante 2.5 meses, corsé de Milwakee y fisioterapia. Posteriormente a] tratamiento se controlaron creatininemia, hemogra-

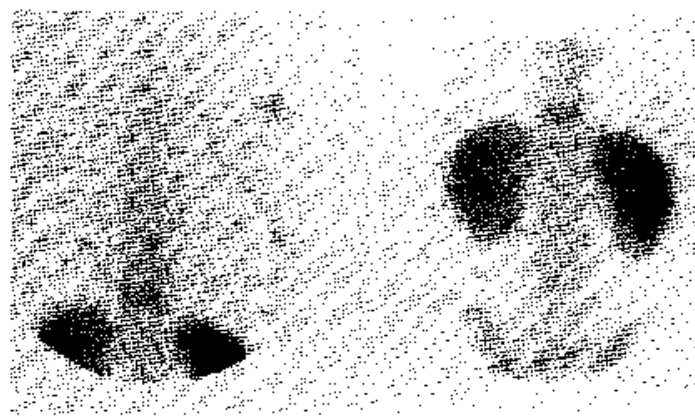

Figura 2: Cintigrafía ósea trifásica que muestra mayor captación a nivel de T10 y T11

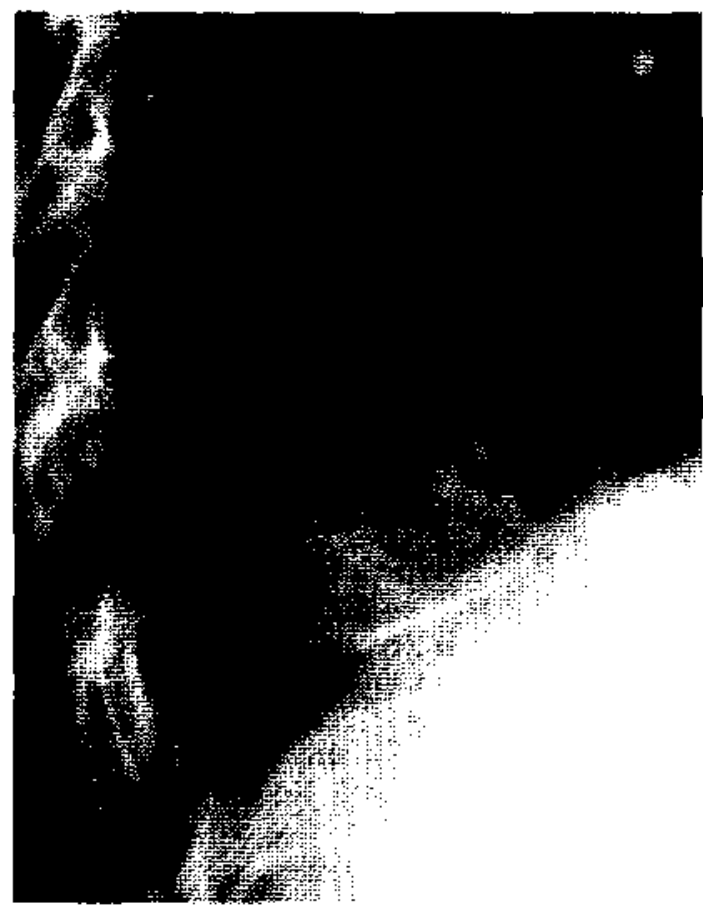

Figura 3: Radiografía de columna lumbar mostrando impactación del cuerpo vertcbral T10 sobre T11.

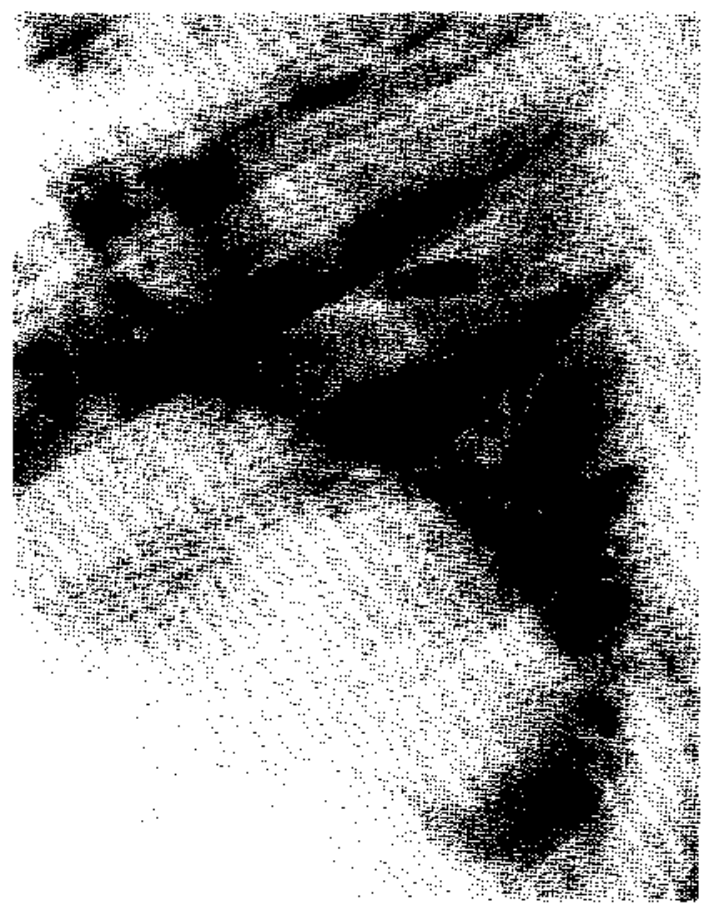

Figura 4: Radiogratía de columna lumbar de control ( 6 meses) que muestra la persistencia de alteraciones a nivel de $T 10$ y $T 11$. 
ma, calcemia, fosfemia, magnesemia, transaminasas y sedimento urinario. El estudio anatomopatológico de una biopsia por punción de Tl1, con el paciente en tratamiento, demostró inflamación inespecífica y el cultívo para hongos fue negativo. Recibió ketoconazol oral durante seis meses. En noviembre de 1990, más de dos años después de su egreso del hospital, estaba asintomático, su crecimiento pondoestatural era ade. cuado, tenía como secuela en el pie derecho una deformación en equino moderado $y$ semirrígido $y$ en la columna leve xifosis indolora de T10 a T11. La onicomicosis ha mejorado con el tratamiento local. Las radiografías mucstran osteopenia intensa de tobillo y tarso del pie deracho, estrechamiento tibiotarsiano, impactación de T4-T5 y T10-T11, escasa esclerosis ósea y de elementos de fusión significativos. Nuevas pruebas de hipersensibilidad retardada muestran anergia selectiva pasa Candida albicans y tricophyton.

\section{Comentario}

En este paciente el estudio de inmunidad celular sólo demostró anergia selectiva, marcada y persistente para el género Candida, que fue interpretada como una forma de deficiencia selectiva de la inmunidad celular, lo hace diferente de otros con estados de inmunosupresión que favorecen $\mathrm{de}$ infecciones micóticas ${ }^{1-4}$.

Los discos intervertebrales son, en los niños, proclives a la localización de infecciones, incluso por hongos. El tratamiento asociado con anfotericina B y 5-fluorcitosina es el indicado en procesos micóticos profundos, permite disminuir las dosis de anfotericina B y minimizar los efectos adversos. E1 resultado del tratamiento fue el esperados. La rehabilitación en estos casos es de gran importancia, por el riesgo de secuelas neurológicas, funcionales y estéticas de la destrucción de discos vertebrales.

\section{Resumen}

Un escolar de 13 años de edad, con antecedentes de candidiasis mucocutánea desde los seis meses de vida, sufrió artritis del tobillo derecho - de cuya biopsia se identificó Candida albicans- y espondilodiscitis de T10-T11 y T4-T5. Se trató con anfotericina B y 5-fluorcitosina durante aproximadamente dos meses y luego con ketoconazol oral por seis meses, sin complicaciones debidas al tratamínto $y$ con negativización de los cultivos para hongos. En la búsqueda de compromiso de su sistema inmunitario se descartaron neoplasias e inmunodeficiencia adquirida (HIV); en cambio se demostró que tenía una deficiencia selectiva de la inmunidad celular para el género Candida $s p$. La rehabilitación iniciada precozmente es fundamental en la prevención de secuelas neurológicas y osteoarticulares en estos pacientes.

(Palabras clave: candidiasis mucocutánea, osteoartritis, espondilodiscitis.)

\section{Agradecimientos}

A Marcelo Wolf, MC, por su colaboración en el manejo antimicótico del pacjente.

\section{Referenciss}

1. Shaikh BS, Appelhatu PC, Aber RC. Vertebral disk Space infection and osteomyelitis due to Candida albicans in patient with acute myelomonocytic leukemia. Cancer 1980; 45: 1025. 1028 .

2. Edwards JE Jr, Turkel SB, Elder HA, Rand RW, Guze LB: Hematogenous candida osteomyelitis: report of three cases and review of literature. Am J Med 1975; 59: 89-94.

3. Nieto $A$, Navarro $V$, Calvo $J$, De la Cuadra $J$. Vicent $R$, Feryer $C$ : Osteomielitis vertebral por Candido albicans en una paciente heroinómana. Rev Esp Reumatol 1987; 14: 57-60.

4. Kunstmann $G$, Rencoret $G$ : Candidiasis sistémica neonatal. Rev Chil Pediatr 1987; 58 : 239-241.

5. Schreiber J, Maynard $E$, Lew $M$ : Candida antigen detection in two prematures neonates with disseminated candidiasis. Pedjatrics; 1984; 74 : 838841.

6. Etwapds JE Jr, Lehrer RI. Stiehm ER, Fisher $T J$. Young LS: Severe candidal infections. Clinical perspective. Immune defense mecharisms and current concepts of therapy. Ann Int Med 1978; 89: 91-106.

7. Simpson MB Jr, Merz WG, Kurtinski JP, Solomon MH: Opportunistic mycotic osteamyelitis: Bone infection due to Aspergillus and Candida species. Medicine 1977; $56: 475-482$.

8. Terrel $C H$. Hermans $P$; Antifungal agents used for deep-seated mycotic infections. Mayo Clin Proc 1987; 62: 1116-1128. 